\title{
Examination of a Proposed Validation Data Set Using CFD Calculations
}

\section{ASME 2009 Fluids Engineering Summer Meeting}

Richard W. Johnson

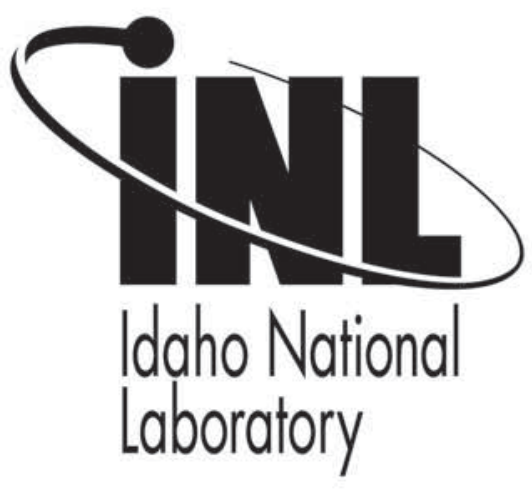

This is a preprint of a paper intended for publication in a journal or proceedings. Since changes may be made before publication, this preprint should not be cited or reproduced without permission of the author. This document was prepared as an account of work sponsored by an agency of the United States Government. Neither the United States Government nor any agency thereof, or any of their employees, makes any warranty, expressed or implied, or assumes any legal liability or responsibility for any third party's use, or the results of such use, of any information, apparatus, product or process disclosed in this report, or represents that its use by such third party would not infringe privately owned rights. The views expressed in this paper are not necessarily those of the United States Government or the sponsoring agency. 


\title{
FEDSM2009-78317
}

\section{EXAMINATION OF A PROPOSED VALIDATION DATA SET USING CFD CALCULATIONS}

\author{
Richard W. Johnson \\ Idaho National Laboratory \\ Idaho Falls, Idaho, USA
}

\begin{abstract}
The United States Department of Energy is promoting the resurgence of nuclear power in the U. S. for both electrical power generation and production of process heat required for industrial processes such as the manufacture of hydrogen for use as a fuel in automobiles. The DOE project is called the next generation nuclear plant (NGNP) and is based on a Generation IV reactor concept called the very high temperature reactor (VHTR), which will use helium as the coolant at temperatures ranging from $450{ }^{\circ} \mathrm{C}$ to perhaps $1000{ }^{\circ} \mathrm{C}$. While computational fluid dynamics (CFD) has not been used for past safety analysis for nuclear reactors in the U. S., it is being considered for safety analysis for existing and future reactors. It is fully recognized that CFD simulation codes will have to be validated for flow physics reasonably close to actual fluid dynamic conditions expected in normal and accident operational situations. To this end, experimental data have been obtained in a scaled model of a narrow slice of the lower plenum of a prismatic VHTR. The present article presents new results of CFD examinations of these data to explore potential issues with the geometry, the initial conditions, the flow dynamics and the data needed to fully specify the inlet and boundary conditions; results for several turbulence models are examined. Issues are addressed and recommendations about the data are made.
\end{abstract}

\section{INTRODUCTION}

The heated gas that has traversed the core of the VHTR enters a lower plenum where it mixes and exits the reactor vessel. Figure 1 illustrates a plan view of the VHTR lower plenum showing numerous cylindrical support pillars, locations of jet inlets (various small circles) and the exit duct. There are concerns that the heated helium may create hot spots in the lower plenum of the reactor vessel because of differential heating of the helium in the core. There is also concern that the helium will not be sufficiently mixed to attain a uniform temperature as it exits the reactor vessel. The application of a CFD code to characterize the helium flow in the lower plenum requires that the calculations be validated against experimental data that has a close relationship to the flow expected.

Experimental data have been taken using particle image velocimetry (PIV) in the INL's matched index of refraction (MIR) facility for a (1:6.55) scaled model of a narrow section of the lower plenum that is representative of the lower plenum flow [1]. Figures 2 and 3 illustrate plan and isometric views of the scaled model, showing inlet ports, half and full support cylindrical posts and the exit plane. The half cylinders are included in the scaled model because the model was designed to be an exact scaled replica of a slice of the lower plenum. The scaled model is $53.98 \mathrm{~mm}$ wide, $558.8 \mathrm{~mm}$ long with the first jet located at $88.93 \mathrm{~mm}$ from the origin, and $217.5 \mathrm{~mm}$ high. The origin is pictured in Fig. 3. Diameters for the inlet jets and posts are $0.0221 \mathrm{~mm}$ and $0.03175 \mathrm{~mm}$, respectively.

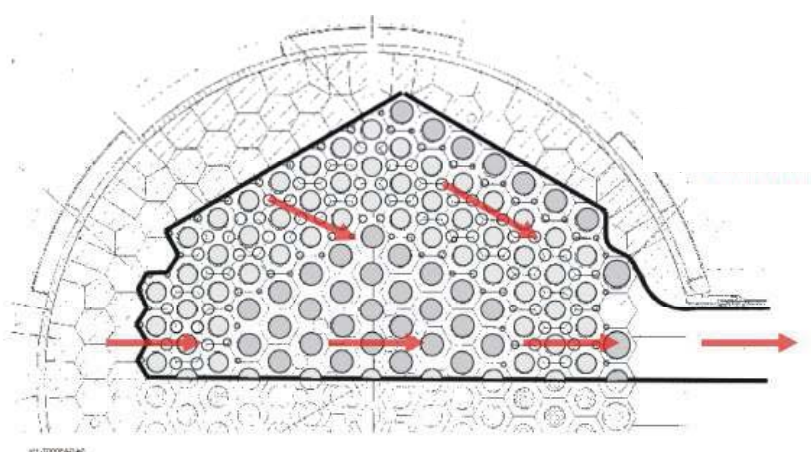

Figure 1. Plan view of the VHTR lower plenum.

Inlet jets (open)

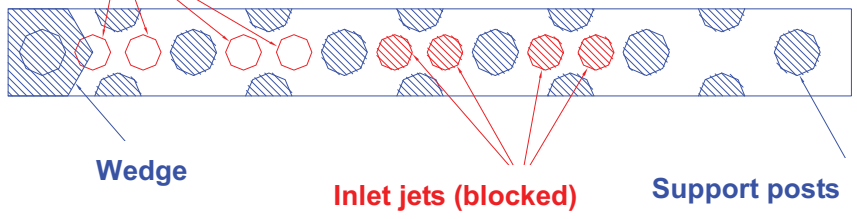

Figure 2. Plan view of the scaled model. 


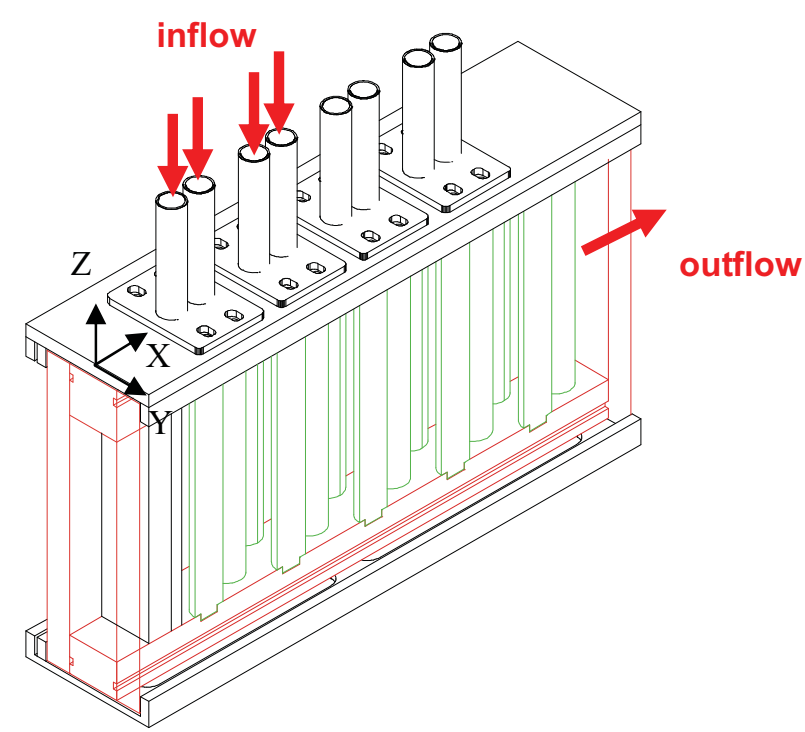

Figure 3. Isometric view of the scaled model.

The scaled model was positioned in the INL's MIR facility test section such that the working fluid (mineral oil) not only flowed through the model, but also around the outside of the model. Previous CFD investigations by the author [2] include the development of an analysis plan to determine whether the outer flow should be included in the computational domain of the CFD model. It was determined that the CFD model need only include the flow inside the model when a 'pressure-outlet' outlet boundary condition is used. This boundary condition, present in both FLUENT [3] and STARCCM+ [4] commercial CFD codes, sets the outlet pressure to a constant. While this condition is not exactly correct, it apparently is close enough to reality such that only the computed flow close to the outlet plane is affected [2]. The analysis plan results were based on two-dimensional flows that mimicked the plan view of the scaled model and employed the unsteady Reynolds-averaged Navier-Stokes equations, called a URANS approach. It was seen that vortex-shedding occurred behind the center full posts as well as the half posts attached to the side walls, making the 2-D turbulent flow nonstationary.

In a subsequent study [5], the author performed 3-D URANS calculations of the scaled model and compared the results to the MIR test data. Based on an earlier CFD study of flow in a staggered tube bank [6], the 3-D calculations employed the Reynolds stress model (RSM) of turbulence. The RSM is the most sophisticated RANS turbulence model available in the commercial codes FLUENT and STARCCM+; it solves a differential equation for each unique Reynolds stress.

Calculations reported in Ref. [5] were made for both uniform inlet conditions (based on separate MIR inlet mass flow measurements) and inlet conditions for turbulent kinetic energy $(k)$ and mean velocity components that were measured in the MIR at about $10 \mathrm{~mm}$ above the top plane of the scaled model. The inlet turbulent dissipation rate was set to a constant.
The RSM URANS calculations decayed to a steady state for the MIR inlet data case and to nearly a steady state for the uniform inlet case. Comparisons between the calculations and the data indicated that the simulations were poor near the first two inlet jets, especially the first jet (which can be considered to be a wall jet and whose center is located at the point of the wedge; see Fig. 2). Beyond the inlet jets, the comparisons are fairly good [5]. The major discrepancy is apparently because the size of the recirculation zone that occurs in the bottom corners of the inlet end of the scaled model is predicted larger than shown in the data. That is, the first jet detaches from the walls of the wedge higher than it should be as it is swept downstream. Also, even though the scaled model is symmetric about a vertical plane that includes the X-axis, both the MIR data and the calculations of Ref. [5] exhibit asymmetries in places; some of the asymmetries in the calculations follow those of the data, while others do not.

The objectives of the present study are to investigate the effects of using RANS turbulence models on the calculations, particularly the effects on the first inlet jet and the associated recirculation region; effects of different initial conditions; also the effects of using inlet conditions based on the MIR inlet data, but modified to reflect the total mass inflow measured by the rotameters at the MIR facility. Further, other potential issues raised while performing the CFD calculations and recommendations regarding the MIR data are addressed. The calculations are compared to the MIR data for a jet inlet Reynolds number $=\bar{u} d_{j e t} / v=12,400$, where $\bar{u}$ is the bulk velocity of the jet.

\section{CFD MODELS}

The earlier study by the author [2] investigated the grid independence of a related 2-D geometry using three grids. It was found that the intermediate and fine grid results were very close. The subsequent study employed a 3-D grid based on the fineness of the intermediate grid, which had 14 million cells. For the present study, a 3-D grid based on the coarsest grid, with 80 cells in the vertical coordinate, is used to see if differentiations can be made among the several variations in initial and inlet conditions and turbulence model. Comparisons will later be made with the intermediate grid, which has 125 cells in the vertical coordinate, to see what the effects of the coarse grid are. Also, other inlet conditions have been extracted from the MIR data at $11 \mathrm{~mm}$ above the top plane of the scaled model. Hence, a new 3-D coarse grid was constructed with inlet planes at $Z=11 \mathrm{~mm}$. This grid is called grid 'a3.' The previous intermediate 3-D grid, with inlet planes at approximately $\mathrm{Z}=9.7 \mathrm{~mm}$, is called grid ' $\mathrm{b} 2$.' Figure 4 illustrates a portion of grid ' $a 3$ ' at the top plane of the CFD model, showing the first two jet inlets and some of the cylindrical posts.

Commercial code STARCCM+ [4] is used for the present calculations. Second-order discretization is used for both space and time; time-stepping is implicit. The time step used for grid a3 is $2.0 \times 10^{-4}$ sec.; the typical time scale of vortex shedding in the present calculations is $\geq 0.03$ seconds, providing at least 100 
time steps per typical vortex event. The flow is isothermal, as is required by the nature of the matching of the indices of refraction in the MIR facility.

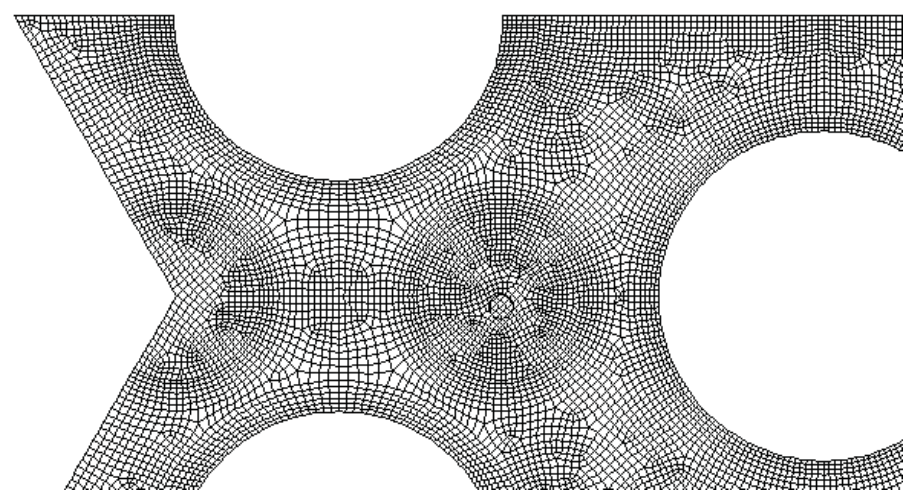

Figure 4. Closeup of grid 'a3.'

Dimensionless wall $y^{+}$values (for wall-adjacent cells) range from about 1 to 12 for most places. The 'pressure-outlet' boundary condition is used at the outlet boundary as discussed earlier. The inlets are either specified as uniform values of mean velocity, turbulent kinetic energy and dissipation rate or are based on MIR measured values of mean velocity and turbulent kinetic energy and constant dissipation rate. For the uniform case, the mean inlet velocity is set at the values measured by mass flow rotameters connected to each jet inlet supply.

The mass flow rates for the MIR inlet data at $Z=11 \mathrm{~mm}$ were examined. It was found that the mass flow rates computed by STARCCM+ for each inlet, using the detailed PIV data, were less than those measured by the individual rotameters by $18.1 \%, 0.78 \%, 3.0 \%$ and $8.8 \%$ for jets $1-4$, respectively; the overall mass flow was $6.7 \%$ low. While each jet profile could be multiplied by a specific factor to obtain the same mass flows as each rotameter, it seems that this approach becomes too far removed from using the actual MIR data. Therefore, the inlet vertical velocity data points are all multiplied by the same factor to yield the same total inlet mass flow rate as for the uniform inlet case. Figure 5 compares the mass flows measured by the rotameters, those calculated using the detailed PIV data and the modified PIV data used for the MIR inlet data cases.

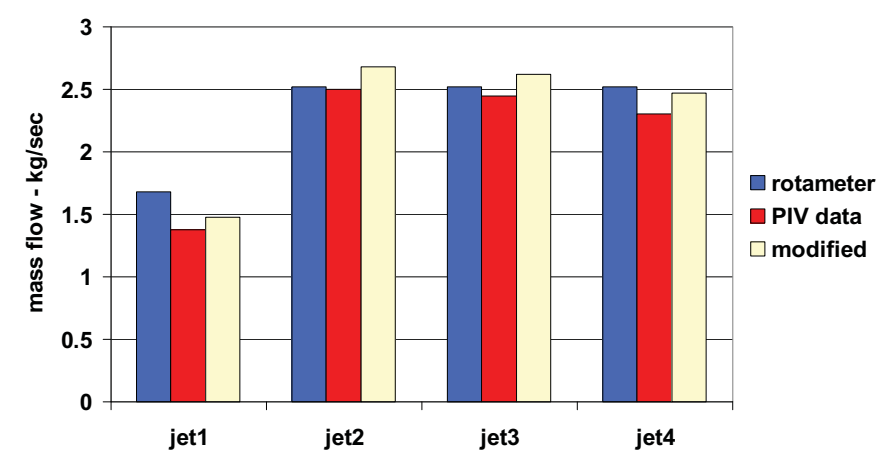

Figure 5. Jet mass flows derived from rotameter and detailed PIV data compared to the modified PIV data.
Initial conditions are varied. In some cases, a steady case is calculated until convergence no longer improves and then the unsteady calculations are begun. In other cases, the unsteady calculation is commenced from a stagnant condition (zero velocity and pressure except at the inlets). Four different turbulence models are employed in the present calculations.

\section{RESULTS AND DISCUSSION}

Results have been obtained for three different two-equation eddy viscosity turbulence models available in STARCCM+ [4]. These are the standard $k \sim \varepsilon$ two layer all $y^{+}$model, the AbeKondoh-Nagano (AKN) $k \sim \varepsilon$ low Re all $y^{+}$model and the Menter shear-stress transport (SST) $k \sim \omega$ all $y^{+}$model. The latter two models are specifically designed to apply to within close proximity of the wall $\left(y^{+}<11\right)$, while the first model breaks the wall region into two layers, a viscous sublayer and a turbulent sublayer. However, the range of $y^{+}$puts the first nodes in the viscous sublayer. Turbulence inlet conditions are set to the default values of turbulence intensity $=0.01$ and turbulent viscosity ratio $=\mu_{t} / \mu=10$. Inlet mean vertical velocities are set to the bulk values measured from the mass flow rotameters. The initial conditions are set to mean velocities of $(1.0,0.0,0.0)$ $\mathrm{m} / \mathrm{sec}$. Each case is computed as a steady state problem until the residuals do not reduce any further. The calculations are then changed to unsteady. All of these cases maintain unsteady conditions throughout the rest of the computation time. Figure 6 shows the streamline pattern computed for the AKN low Re turbulence model at 3.14 seconds.

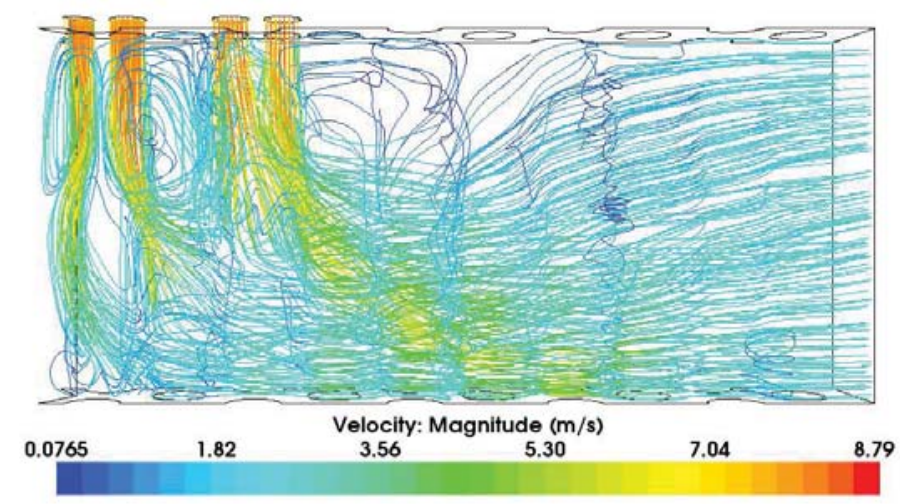

\section{Figure 6. 3-D Streamlines for AKN model at $3.14 \mathrm{sec}$.}

It can be seen that there are recirculation zones near the first jet (toward the left) at the top and at the bottom, between jets 2 and 3 and a large low flow zone at the upper center of the domain. These correspond to similar recirculation zones that appear in a flow visualization experiment performed at the MIR [5].

Figure 7 illustrates profile locations in the scaled model that will be referred to for data comparisons. The profiles shown in the plan view each represent two profiles: one at $Z=-70 \mathrm{~mm}$ (given suffix ' $u$ ') and one at $Z=-150 \mathrm{~mm}$ (suffix 'd'). So for 
example, profile ' $x 1 \mathrm{u}$ ' is at location ' $\mathrm{x} 1$ ' at $\mathrm{Z}=-70 \mathrm{~mm}$, while 'p5d' is at point $\mathrm{p} 5$ at $Z=-150 \mathrm{~mm}$.

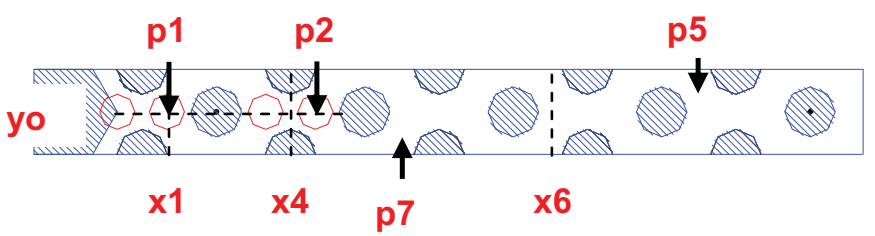

Figure 7. Locations of profiles for data comparison.

For the present case of nonstationary turbulent flow, two averaging operations can be applied to the instantaneous flow quantities [6]. First, the random turbulence is averaged out by performing an ensemble average. Conceptually, this is done by considering that the flow is repeated many times and that the ensemble of results is simply averaged. Mathematically, the instantaneous flow quantities are decomposed into an ensemble average and a (random) turbulent fluctuating component. For example, for the $\mathrm{X}$-component velocity:

$$
u=\langle u\rangle+u^{\prime}
$$

where $\langle u\rangle$ is the ensemble average and $u^{\prime}$ is the turbulent fluctuation. The ensemble average can vary in time because of non-turbulent unsteadiness. The ensemble-averaged quantity can be decomposed into a time-averaged component and a coherent fluctuation. The coherent fluctuation is related to nonturbulent unsteadiness such as vortex-shedding. For example

$$
<u>=U+\tilde{u}
$$

where $U$ is the time average of the ensemble average (or longtime average or just time mean) and $\tilde{u}$ is the coherent fluctuation. These averaging processes are applied to the velocity components and pressure. The velocity components for the present study are $u, v$ and $w$ in the $\mathrm{X}, \mathrm{Y}$ and $\mathrm{Z}$ directions. The CFD calculations presented herein compute the ensemble averages. These are also time-averaged (by a running timeaveraging calculation) because the experimental data [1] are long-time averaged. Furthermore, the data [1] were taken at a rate of 2 - 3 data planes per second, which is insufficiently rapid to capture the vortex shedding. However, the data [1] were taken for time intervals exceeding 300 seconds such that a fairly representative long-time average should have been obtained.

Figures 8,9 and 10 plot time traces for the ensemble-mean $\mathrm{X}$-velocity $\langle u\rangle$ at points $\mathrm{p} 5 \mathrm{~d}, \mathrm{p} 7 \mathrm{~d}$ and $\mathrm{p} 7 \mathrm{u}$ for turbulence models: standard $k \sim \varepsilon$; AKN $k \sim \varepsilon$; and SST $k \sim \omega$, respectively. Unlike results reported using the Reynolds stress transport model (RSM) [5], these results all indicate nonstationary flow.

The standard $k \sim \varepsilon$ two layer traces are all very regular, while those for the other two models are very irregular, with oscillation amplitudes being greatest for SST $k \sim \omega$ and least for the standard $k \sim \varepsilon$ model. This is indicative of the overall level of turbulence viscosity, that is, highest for the lowest amplitude oscillation. The irregularity of the oscillations in Figs. $9 \& 10$ is likely because of passing shed vortices from upstream, each with somewhat different shedding frequency. As shown in Figs. 8 - 10 , the typical vortex shedding time scale is $\geq 0.03 \mathrm{sec}$.

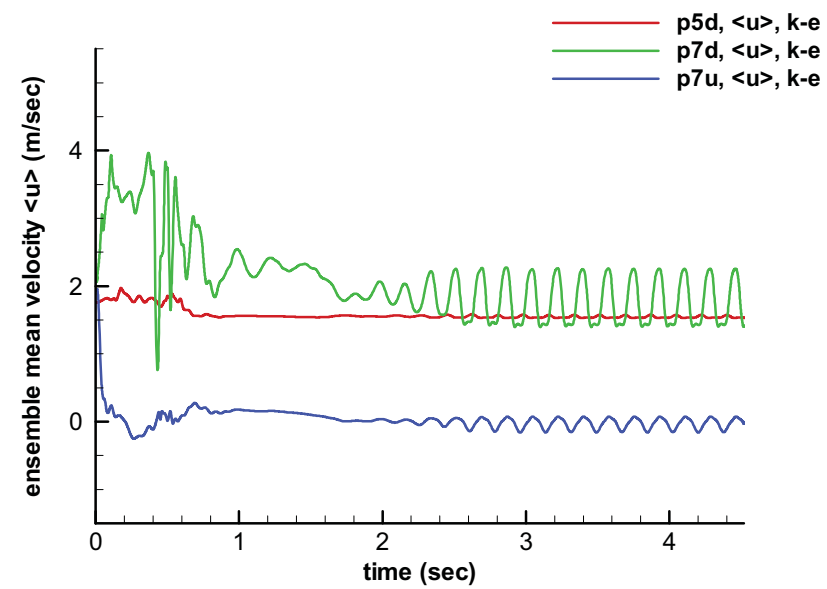

Figure 8. Time traces of the ensemble mean velocity $<u>$ for the standard $k \sim \varepsilon$ model.

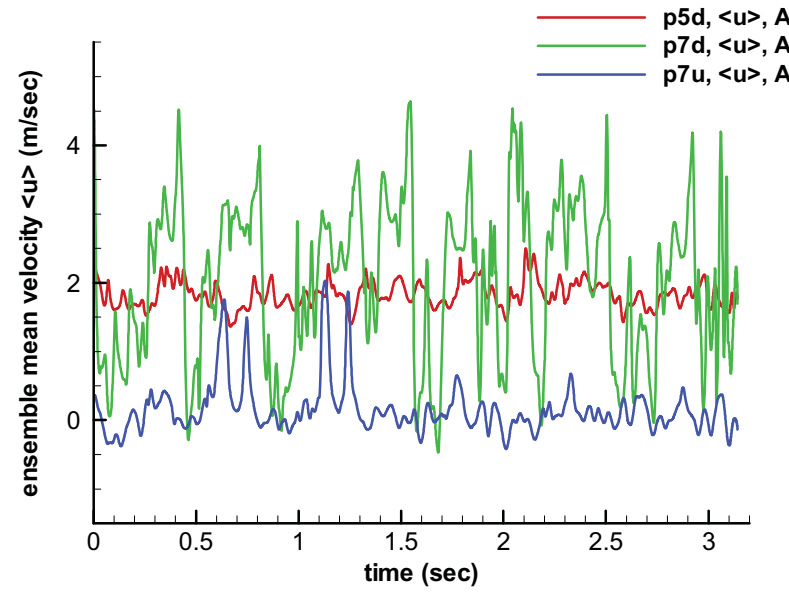

Figure 9. Time traces of the ensemble mean velocity $<u>$ for the AKN $k \sim \varepsilon$ model.

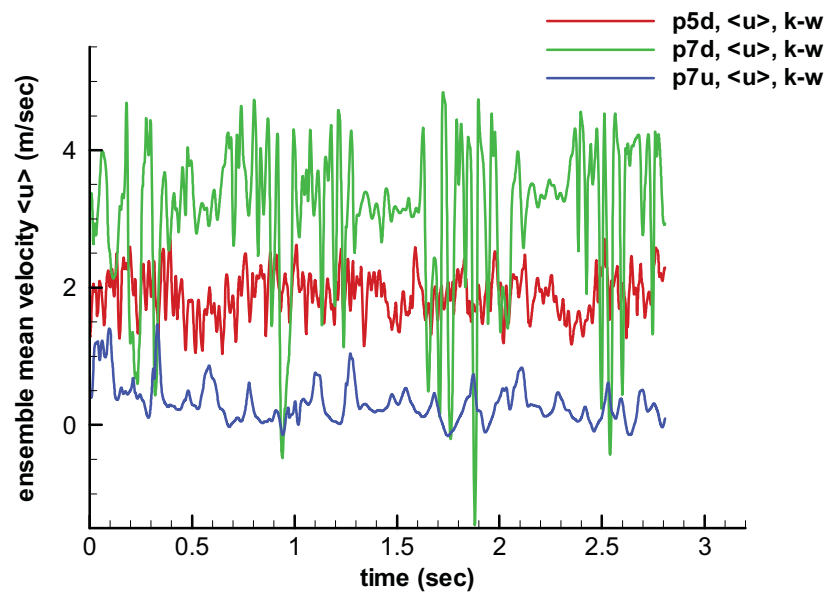

Figure 10. Time traces of the ensemble mean velocity $<u>$ for the SST $k \sim \omega$ model.

The turbulent time scale for the large turbulent eddies is $\tau \sim 1 / \omega$, where $\omega$ is the specific dissipation of the $k \sim \omega$ model [4]. For the present flow, the large turbulence time scale is of the order of 
$0.003 \mathrm{sec}$. This represents a separation of an order of magnitude between turbulence and vortex-shedding time scales.

The anomalous spikes that can be seen for p7u in Fig. 9 and for p7d in Fig. 10 may simply be the infrequent coincidence of shed vortices that sum either amplitude peaks or troughs. The traces for point $\mathrm{p} 7 \mathrm{~d}$ exhibit the largest amplitude oscillations for all models. Additionally, the apparent long-time averages for point $\mathrm{p} 7 \mathrm{~d}$ are all rather different for the three models, while those for the other points are much closer. These results imply that there are some large variations in the flow dynamics in the flow near the inlet jets.

Figures 11 and 12 illustrate profiles for the time mean velocity $W$ at 'you' and 'yod' locations for the three turbulence models. Velocities for the standard $\mathrm{k} \sim \varepsilon$ model are averaged over 4 cycles. Velocities are averaged over the whole time for the other two models. At 'you', the signatures of the four jets are clearly visible in the MIR data. However, for the standard $k \sim \varepsilon$ model, the first jet is missing. Results for the other two models clearly show the first jet, albeit with lower magnitude than the data. The location of the second jet is also somewhat off for the three models, while the third and fourth jets are predicted in the right locations, again with somewhat too low magnitude. At 'yod', the $W$ velocity profile is not well predicted by any of the

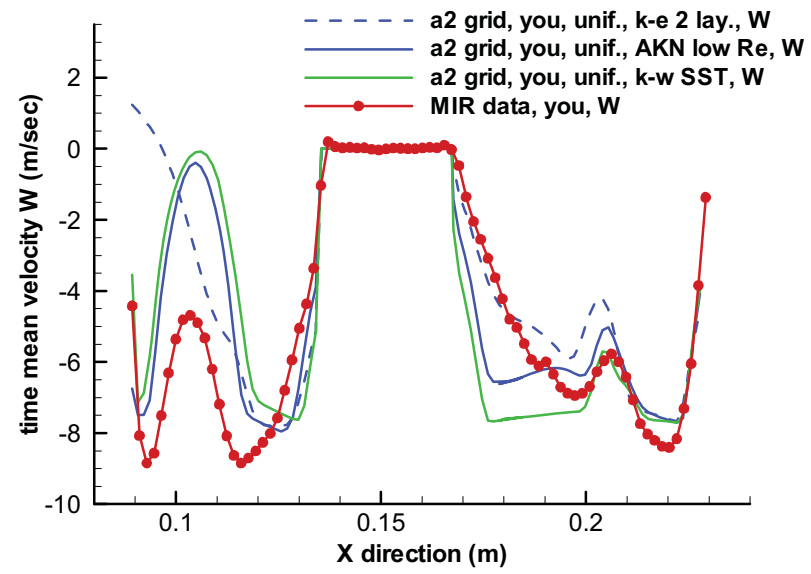

Figure 11. Time mean velocity $W$ at 'you.'

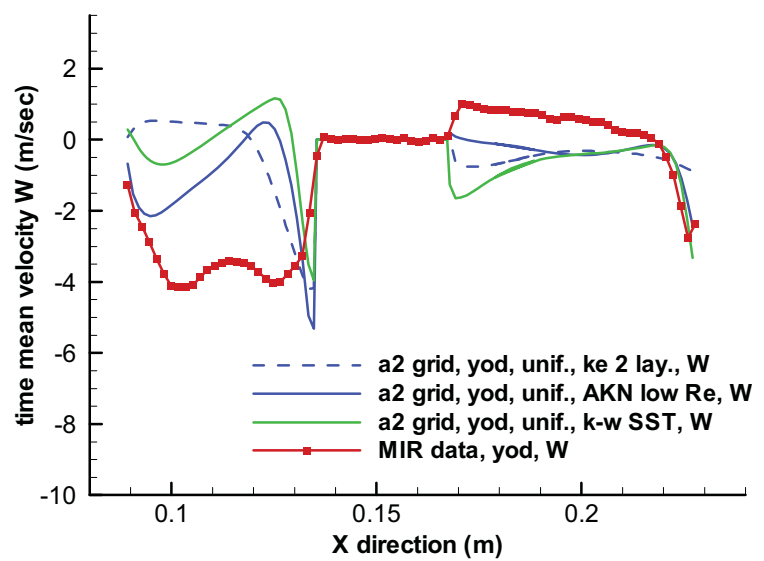

Figure 12. Time mean velocity $W$ at 'yod.' models, though the predictions for the AKN $k \sim \varepsilon$ model are best. In particular, the signature for the first jet is too weak while that for the second jet is too strong; the locations for each are somewhat different from the data. The standard $k \sim \varepsilon$ and $k \sim \omega$ models exhibit a signature for the third jet when they shouldn't'.

Figure 13 illustrates contour plots of time mean velocity $W$ for jet 1 in a plane normal to the X-direction and through the jet center for the standard $k \sim \varepsilon$ model, the AKN $k \sim \varepsilon$ model and the MIR data, all scaled the same. Clearly, the skewing of jet 1 to the side for the standard $k \sim \varepsilon$ model has caused the profile at $Z=-70$ $\mathrm{mm}$, Fig. 11, to miss the signature of the jet, while the AKN $k \sim \varepsilon$ model shows no skewing with jet 1 captured well in Fig. 11. The peak velocity is greater in the MIR data than for the calculations. Results for the SST $k \sim \omega$ model, not shown, are very similar to those for the the AKN $k \sim \varepsilon$ model. Results for the RSM in Ref. [5] are also skewed to the side.

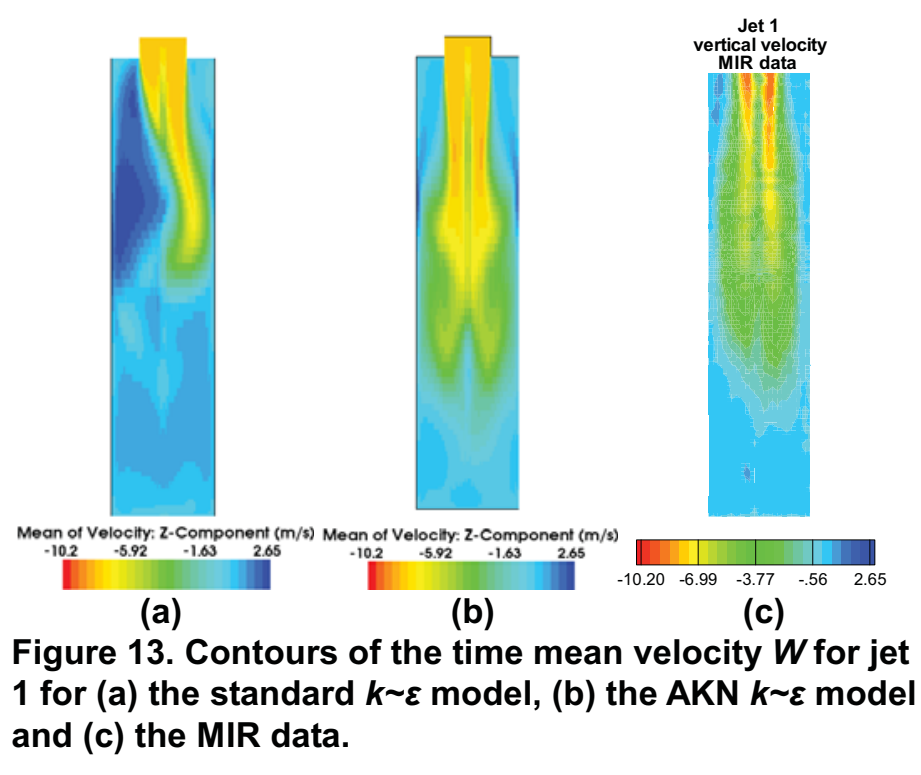

Figure 14 provides contours of the time mean velocity $W$ at a plane located at $\mathrm{Z}=-70 \mathrm{~mm}$ for the standard $k \sim \varepsilon$ model, the AKN $k \sim \varepsilon$ model and the MIR data. (It should be noted that the MIR data do not extend to the outlet of the scaled model because of optical inaccess; also note that the contours levels are all matched.) This view clearly shows how the standard $k \sim \varepsilon$ model results are asymmetrically skewed to one side while those for the AKN $k \sim \varepsilon$ model and the MIR data appear to be symmetric. The jet velocity magnitudes for both sets of calculations, however, are lower than for the MIR data. Interestingly, the 'shapes' of the jet signatures are quite similar for the AKN results and the MIR data. It is also the case that the AKN results mimic the upwards flow of the MIR data in the corners behind the first jet and above and below the first full post. 


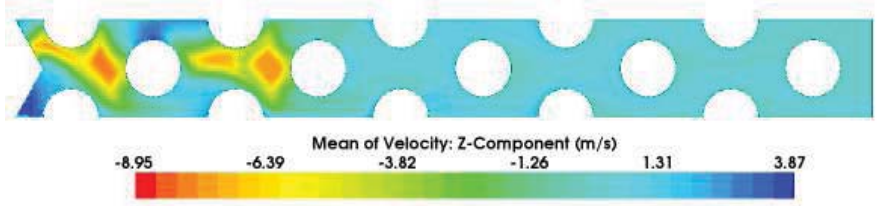

(a)

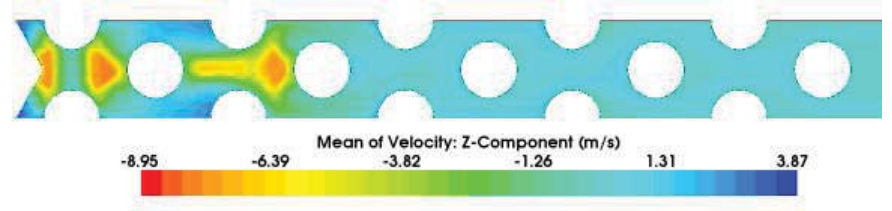

(b)

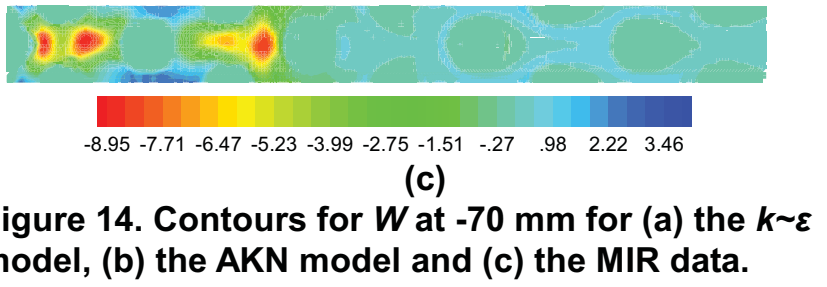

It is remarkable how the difference between the two $k \sim \varepsilon$ models, with all else equal, can provoke a significant departure from symmetry in the flow pattern. As observed in Ref. [5], it appears that subtle variations that may be hard to control or identify can lead to asymmetries. Such asymmetries may be slight variations of the actual geometry from the design geometry, slight variations in the inlet conditions or slight variations in computed results. Another possible explanation for the strange behavior of the asymmetric $k \sim \varepsilon$ model results is that there are multiple potential solutions that may or may not be stable (or symmetric) and that small perturbations may cause the simulation to migrate to one or the other of these solutions. It actually is quite difficult to achieve a high degree of symmetry. This concern argues for a geometry that has built-in asymmetry to over-ride other subtle causes of asymmetry for a validation data set.

Figures $15-17$ plot profiles of time mean velocity $U$ for the standard $k \sim \varepsilon$ two layer; AKN $k \sim \varepsilon$ low Re; and SST $k \sim \omega$ models for profile locations $x 1, x 4$ and $x 6$ at $Z=-70 \mathrm{~mm}$ and $Z=-150$ $\mathrm{mm}$. Profiles for the three turbulence models are quite different from each other and from the data for profiles ' $x 1 \mathrm{u}$ ' and $\mathrm{x} 1 \mathrm{~d}$,' located below the second inlet jet. The AKN results are quite symmetric, while the $k \sim \omega$ results are somewhat asymmetric and the $k \sim \varepsilon$ results are very asymmetric as are the MIR data, though not in the same way. For locations ' $x 4 u$ ' and ' $x 4 d$,' Fig. 16, the calculations show better agreement with the MIR data, except for the standard $k \sim \varepsilon$ model at ' $\mathrm{x} 4 \mathrm{~d}$,' where the trend is dramatically wrong. Beyond the inlet jet region, calculations at 'x6u' and 'x6d,' Fig. 17, show quite reasonable agreement, especially for the AKN $k \sim \varepsilon$ model, which agree quite well with the data. The trend of showing improved agreement for increasing $\mathrm{X}$, especially beyond the inlet jet region, was also found for the RSM in Ref. [5]. The agreement for the AKN model with the MIR data at ' $x 6$ ' is better than for the RSM [5].

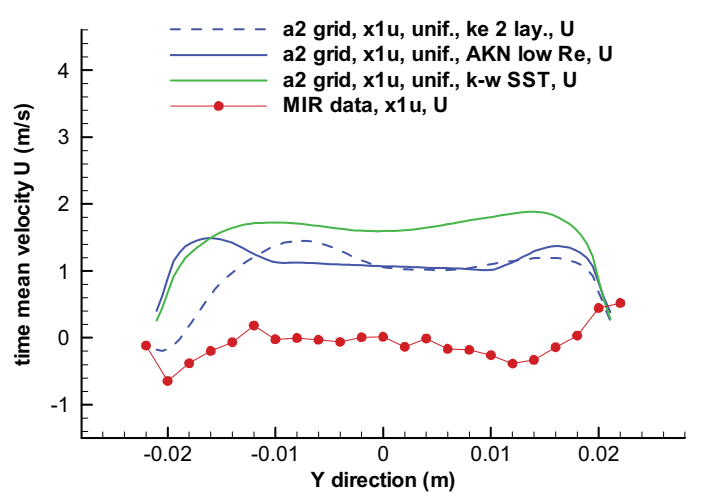

(a)

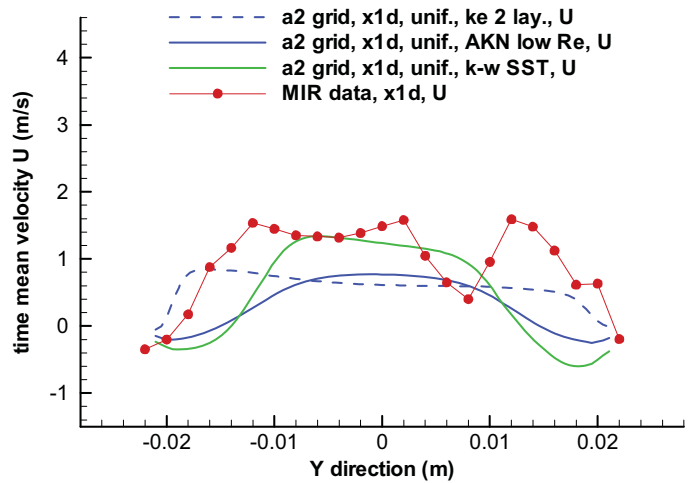

(b)

Figure 15. Time mean velocity $U$ at (a) $\times 1 u$ and (b) $\times 1 d$.

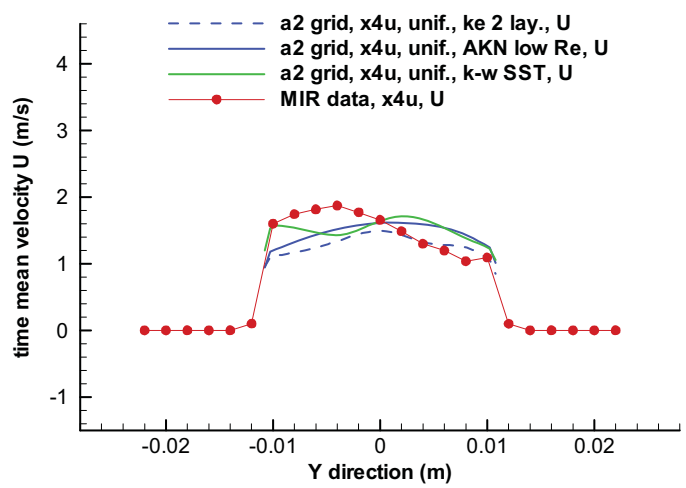

(a)

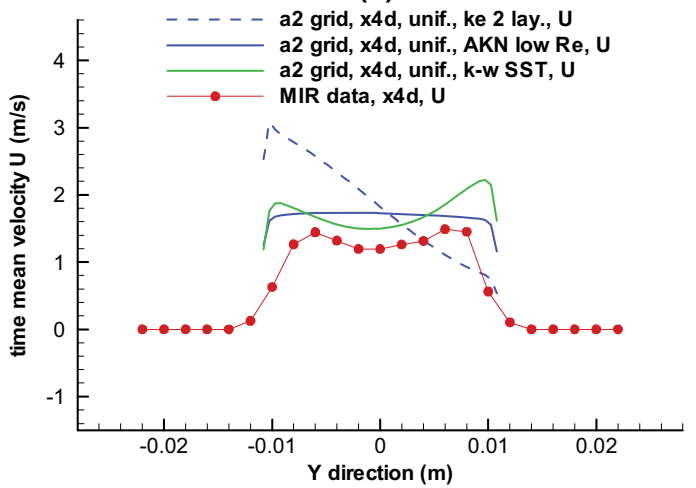

(b)

Figure 16. Time mean velocity $U$ at (a) $x 4 u$ and (b) $x 4 d$. 


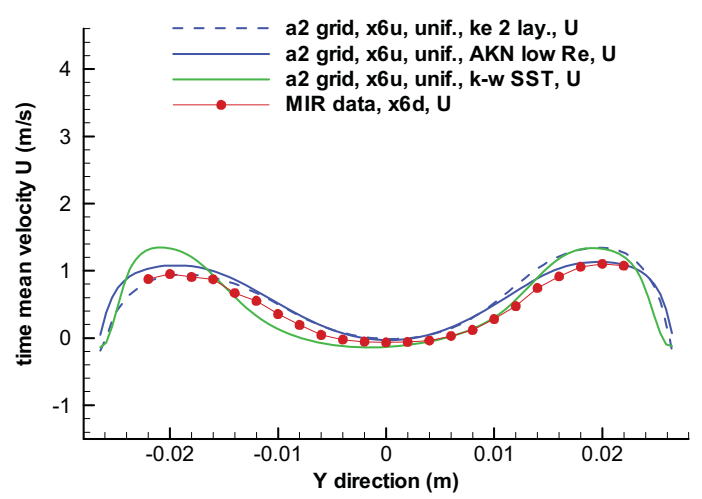

(a)

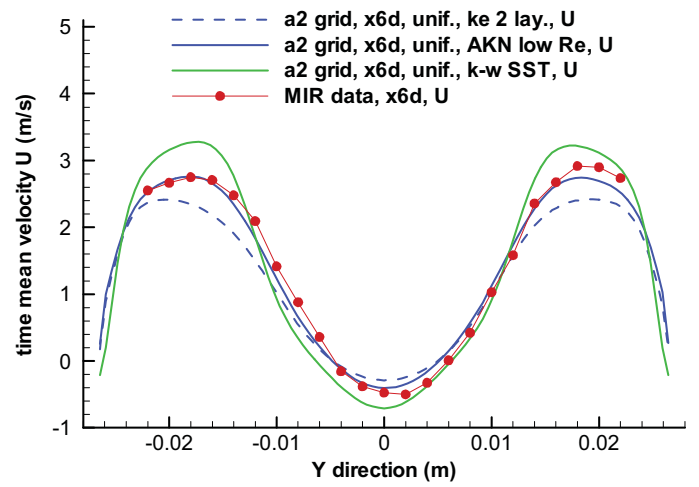

(b)

Figure 17. Time mean velocity $U$ at (a) $\times 6 u$ and (b) $\times 6 \mathrm{~d}$.

Additional computations have been made using different inlet and initial conditions than for the calculations above. These have been applied to the AKN $k \sim \varepsilon$ model and the RSM model with linear pressure strain and high $y^{+}$wall treatment (equivalent to standard wall functions as was used in an earlier study [2]). These models are chosen because of their superior performance over the other models examined. The MIR data at $11 \mathrm{~mm}$ above the plane of the inlet $(Z=0.011 \mathrm{~m})$ have been employed for inlet conditions. The MIR data provide the three long-time averaged velocity components and turbulent kinetic energy. As discussed in detail earlier, the vertical velocity components of all the jets are multiplied by a single constant factor such that STARCCM+ yields the same total mass flow rate as measured by the rotameters and used for the uniform inlet cases above.

The inlet dissipation rates are set to $1.0 \mathrm{~J} / \mathrm{kg}$-sec. Initial conditions for the AKN $k \sim \varepsilon$ model are obtained in the first instance from computing a steady case until convergence is minimal and then starting an unsteady calculation. In the second case, the AKN $k \sim \varepsilon$ model and the RSM are computed from an initial stagnant condition where the velocity components and pressure are all set to zero (with $k=0.1 \mathrm{~J} / \mathrm{kg}$ and $\varepsilon=1.0 \mathrm{~J} / \mathrm{kg}$ $\mathrm{sec})$.

Figures 18-20 show time traces for ensemble mean velocity $\langle u\rangle$ at points $\mathrm{p} 5 \mathrm{~d}$ and $\mathrm{p} 7 \mathrm{u}$ and for ensemble mean velocity $\langle w\rangle$ at $p 1 u$ and $p 2 u$ for the three cases just described.

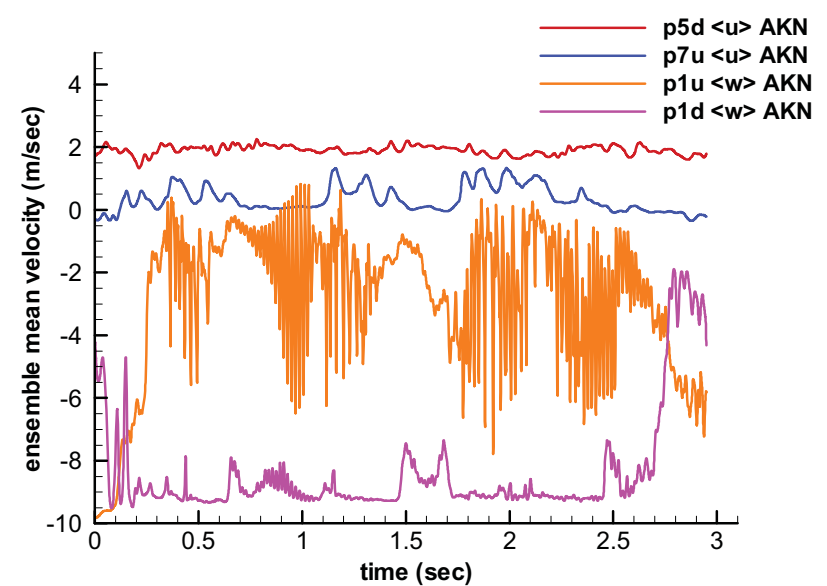

Figure 18. Time traces for $\langle u\rangle$ and $\langle w\rangle$ for AKN $k \sim \varepsilon$ case from steady condition.

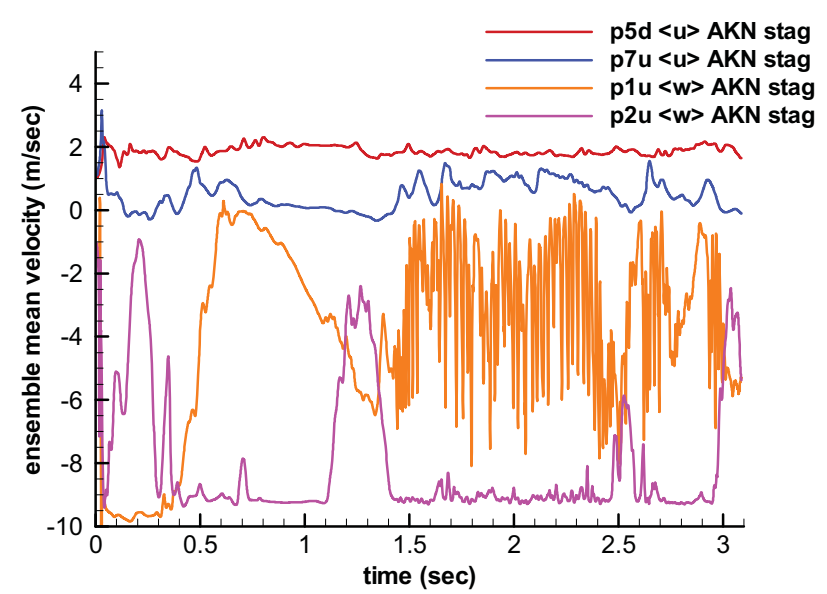

Figure 19. Time traces for $\langle u\rangle$ and $\langle w\rangle$ for AKN $k \sim \varepsilon$ case from stagnant initial conditions.

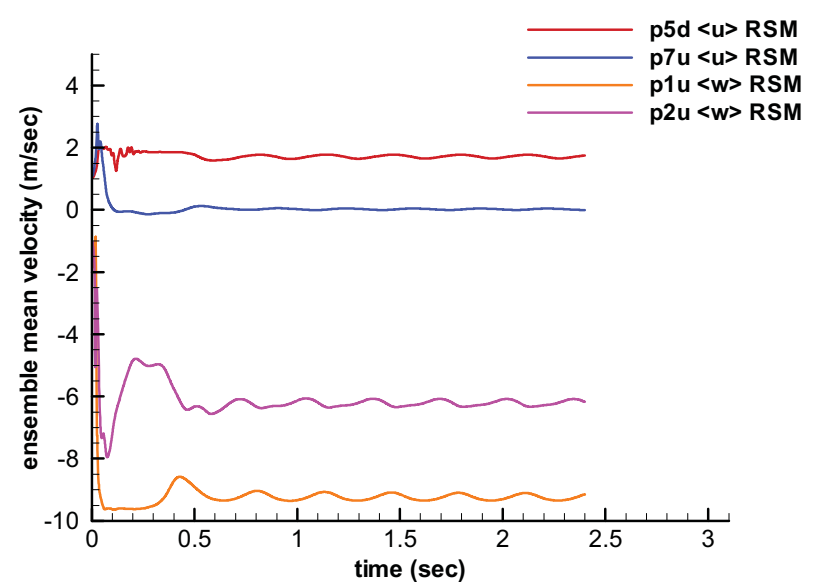

Figure 20. Time traces for $\langle u\rangle$ and $\langle w\rangle$ for RSM case from stagnant initial conditions.

The point locations are given in Fig. 7. Comparing Figs. 18 and 19 , the four time traces exhibit similar characteristics for the two cases. These include low-amplitude oscillations for velocity $\langle u\rangle$ 
at $\mathrm{p} 5 \mathrm{~d}$; a range of large and small scale (time-wise) excursions of $\langle u\rangle$ at $\mathrm{p} 7 \mathrm{u}$; very large amplitude excursions interspersed with small amplitude oscillations for $\langle w\rangle$ at $\mathrm{p} 1 \mathrm{u}$; and dynamic changes in local magnitude of $\langle w\rangle$ at $\mathrm{p} 2 \mathrm{u}$. This latter behavior at $\mathrm{p} 2 \mathrm{u}$ seems to be correlated with the small amplitude oscillations of $\langle w\rangle$ at $\mathrm{plu}$. This appears to be evidence of there being multiple unstable solutions between which the simulations jump over time, particularly in the inlet region. By contrast, the time traces for the RSM model shown in Fig. 20 are very mild in character and appear to be moving toward a steady state solution as was the case for the RSM calculations reported in Ref. [5]. Clearly, the turbulence model has a significant impact on the results for the present problem, not only on the long-time average quantities, but also on the dynamic nature of the simulation.

Figures 21 and 22 show results for time mean velocity $W$ at locations 'you' and 'yod,' for the three cases mentioned. The most obvious shortcoming of the calculations is that the signature of the first jet is missing or nearly missing at both 'you' and 'yod' for all three cases. This situation is at least partly because the mass flow of the first inlet jet is still significantly low (12.2\%) compared to the flow measured by the rotameter, as discussed earlier (Fig. 5). The signature of the fourth jet is correctly captured (by all three cases). At 'yod' the AKN model results exhibit a signature for the third jet, though such is missing in the data. Comparing these results with the previous ones for the AKN model using uniform inlet conditions, besides the lack of a first jet signature, there are some significant variations, indicating that the variation in inlet conditions has a significant impact on the results, as does the turbulence model. Overall, it appears that the RSM results are the best for these three cases. Finally, the differences between the AKN results for different initial conditions are minor compared to the differences relative to the RSM results or to the AKN results using uniform inlet conditions.

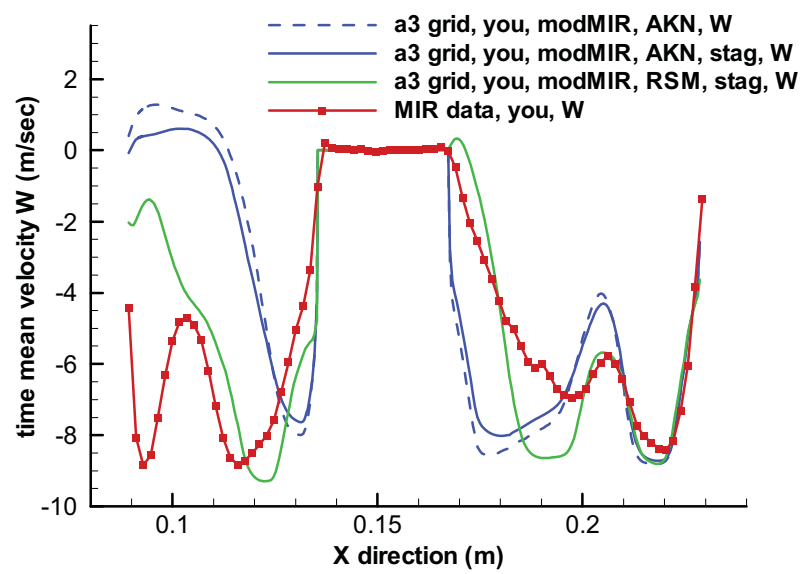

Figure 21. Time mean velocity $W$ at 'you.'

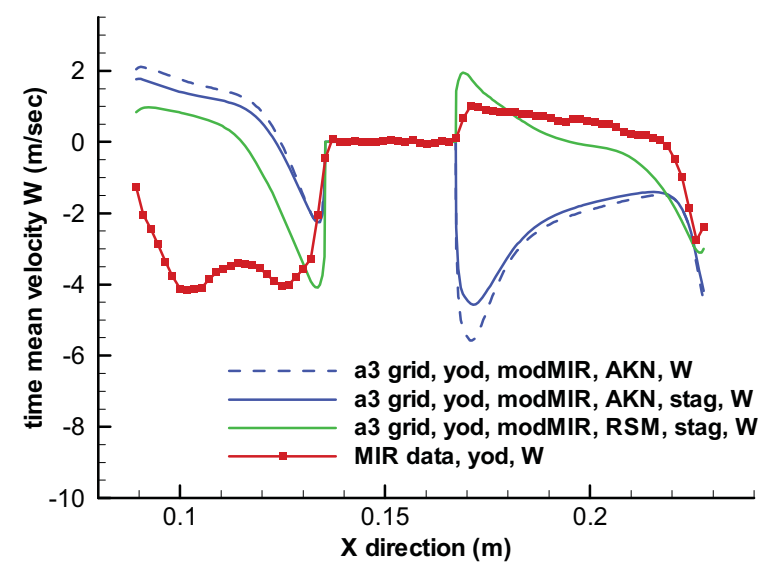

Figure 22. Time mean velocity $W$ at 'yod.'

Additional comparisons are made looking again at contour plots of the time mean velocity $W$ at a plane located at $Z=-70$ $\mathrm{mm}$. Figure 23 shows contours of $W$ for the AKN $k \sim \varepsilon$ model starting from a partially converged steady state condition and for the RSM starting from stagnant conditions. The contours are scaled the same as for Fig. 14 above; the contour for the second jet in Fig. $23 \mathrm{~b}$ is missing because it reaches $-9.37 \mathrm{~m} / \mathrm{sec}$. The RSM results from this view indicate significant asymmetry for the second jet and the flow upstream and for a ways downstream, while the AKN results better match the MIR data, Fig. 14c, and are quite symmetric, though the shapes of the jet signatures are not quite right. Hence, an interesting conundrum is raised: judging by the results plotted in Figs. 21-22, the RSM results appear to be the best, but judging by the results plotted in Fig. 23 , which is at the same $\mathrm{Z}$ location and for the same velocity component, the RSM results are poorer than the AKN results. Actually, the contour plots provide more information about the flow than the profile plots of Figs. 21-22; it appears that the better agreement of the RSM results with the profile plots of Figs. 21-22 may be somewhat fortuitous and that other profiles taken along other values of the $\mathrm{Y}$ coordinate would yield poorer comparisons with the data.

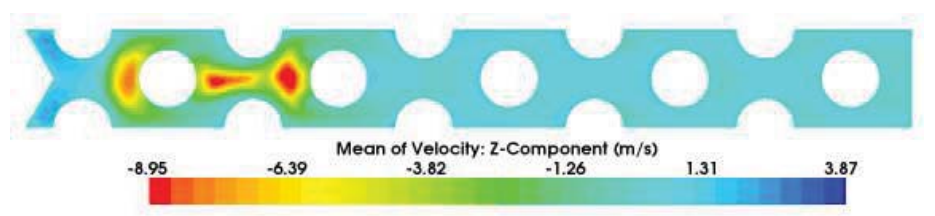

(a)

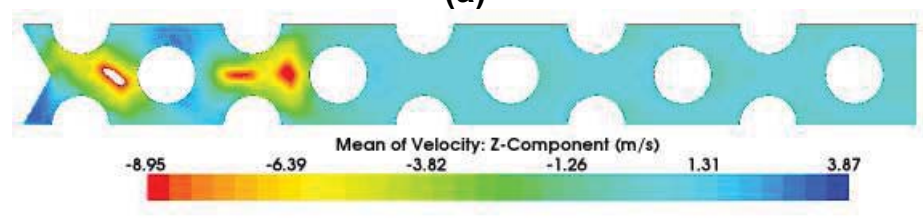

(b)

Figure 23. Contours of time mean velocity $U$ at $Z=-70$ $\mathrm{mm}$ for (a) the AKN model and (b) for the RSM. 
Figures 24-26 plot profiles of time mean velocity $U$ for the three cases: the AKN $k \sim \varepsilon$ model starting from a partially converged steady state, the same model started from stagnant conditions and the RSM started from stagnant conditions. Again, the inlet conditions are modified MIR data at $Z=11 \mathrm{~mm}$ for the three velocity components and the turbulent kinetic energy. In all cases, the results for the two AKN models are sufficiently close that their differences can be explained by the length of time used to compute the averages (and including the initial transient for the stagnant case).

Results are plotted for $x 1 u, x 1 d, x 4 u, x 4 d, x 6 u$ and $x 6 d$ for completeness in comparison with the corresponding profiles shown in Figs. 15-17. The primary difference at ' $x 1$ ' for the AKN results is that $U$ is well-underpredicted at $\mathrm{x} 1 \mathrm{~d}$; The RSM results are rather better than the AKN results. The most interesting differences relative to the earlier results occur at ' $x 4$.' The AKN profiles show asymmetry similar to the data at $\mathrm{x} 4 \mathrm{u}$, as do the RSM results, though the RSM trend is closer to the data, Fig. 25a. For $\mathrm{x} 4 \mathrm{~d}$, the AKN results show the same trend as the data, though the magnitude is too high. The RSM results show inappropriate asymmetry though the magnitude is about right. The calculated profiles at $\times 6 \mathrm{u}$ and $\times 6 \mathrm{~d}$ again show the best agreement with the data and with each other.

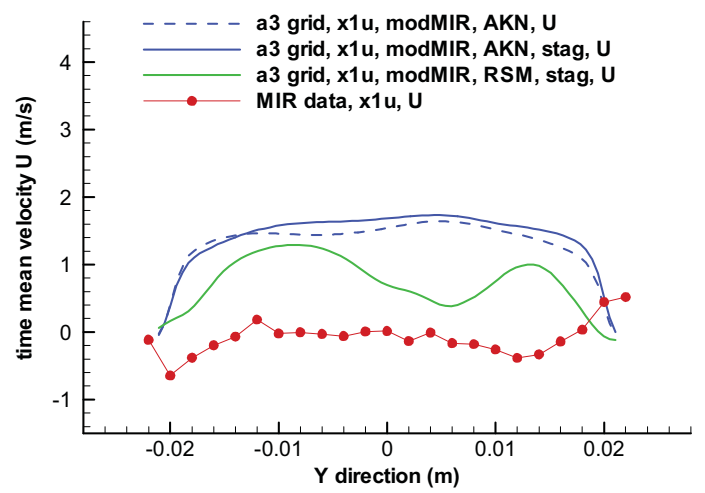

(a)

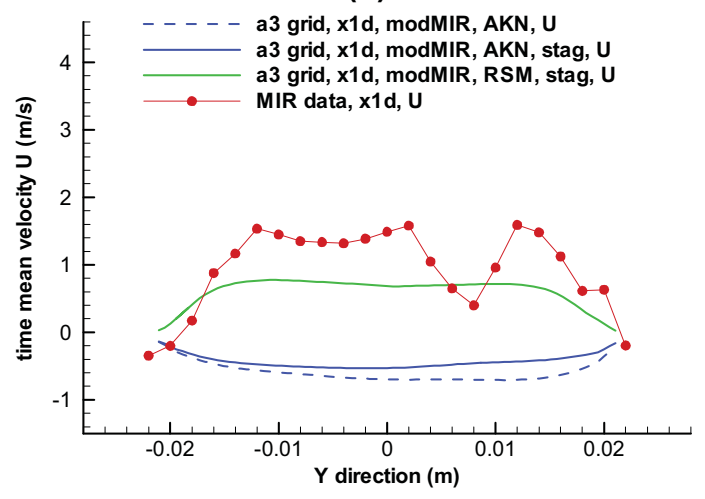

(b)

Figure 24. Time mean velocity $U$ at (a) $x 1 \mathrm{u}$ and (b) $\times 1 \mathrm{~d}$.

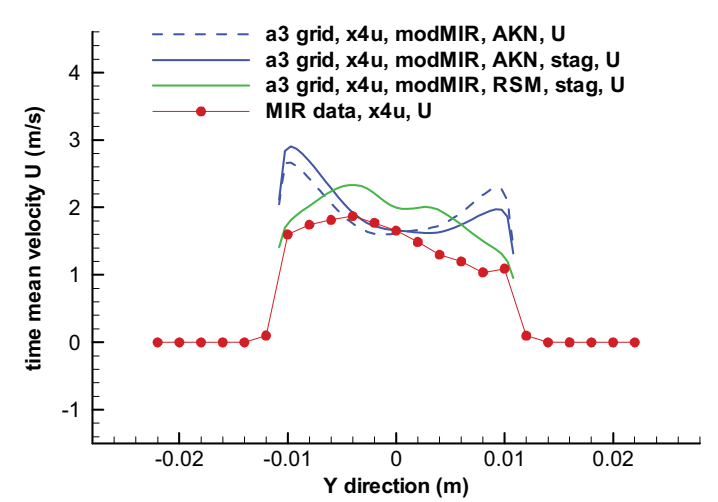

(a)

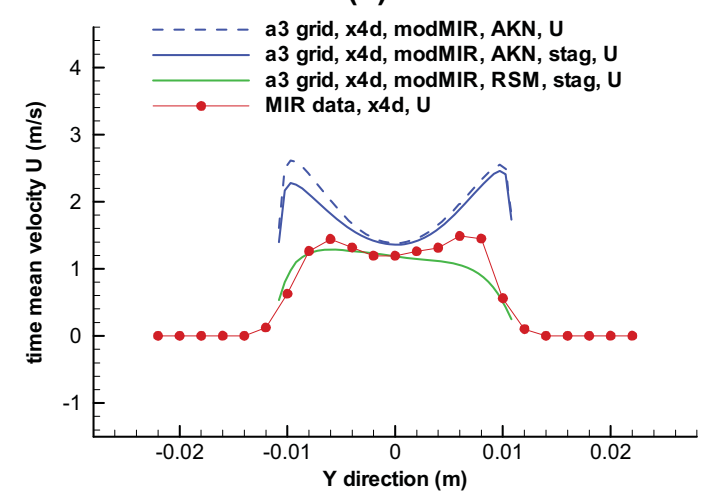

(b)

Figure 25. Time mean velocity $U$ at (a) $x 4 \mathrm{u}$ and (B) $\times 4 d$.

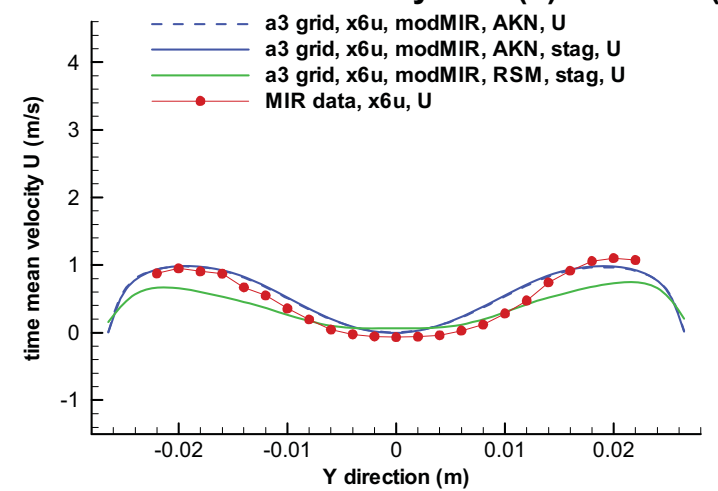

(a)

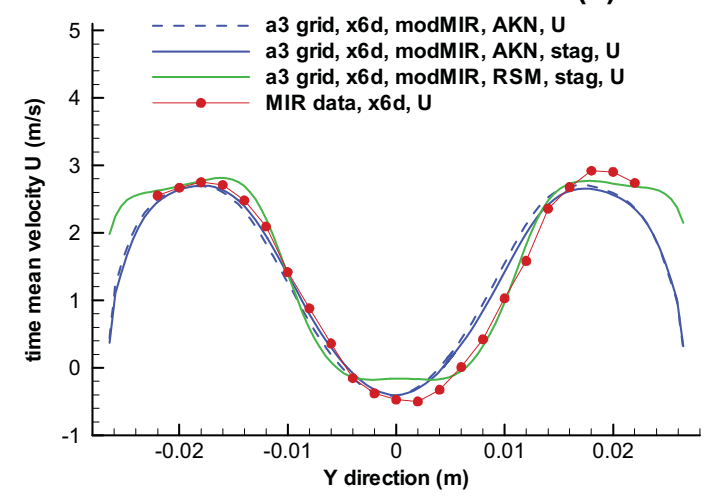

(b)

Figure 26. Time mean velocity $U$ at (a) $x 6 \mathrm{u}$ and (B) $x 6 \mathrm{~d}$. 


\section{SUMMARY AND RECOMMENDATIONS}

In the present article, experimental data taken in the INL's MIR test facility for a scaled section of the lower plenum of the VHTR reactor have been examined; these data were taken with the object of providing a CFD validation data set for CFD calculations to be used in designing and analyzing the VHTR. Several findings have been obtained that relate to a CFD analysis of the flow measured and some recommendations that follow from the examination are made.

Computations for several variations of initial conditions, inlet conditions and turbulence models have been obtained for the flow geometry. Overall, the variation in initial conditions had little effect. Unsteady calculations are begun after a steady state computation is made (and would not converge any further, and is not converged) and from stagnant flow conditions. Inlet conditions used include uniform inlet flow based on mass flow data taken by mass flow rotameters at the MIR facility and detailed velocity component and turbulence kinetic energy data taken in the MIR in the inlet jets above the inlet plane. The vertical velocity component for these data is increased uniformly by about $7 \%$ to match the overall mass flow rate used for the uniform inlet data. The results for the two variations in inlet conditions showed significant variations, especially in the location of the signature of the first inlet jet, even using the same turbulence model. This result is partly attributed to the fact that the modified PIV data have a mass flow for jet 1 that is still $12.2 \%$ below that measured by the mass flow rotameter for jet 1 .

The greatest variation in results is attributable to the turbulence modeling. The four turbulence models used are the standard $k \sim \varepsilon$ two-layer model, the AKN low Re $k \sim \varepsilon$ model and the SST $k \sim \omega$ model all with all $y^{+}$wall treatments; the Reynolds stress transport model (RSM) with linear pressure strain and high $y^{+}$wall treatment is also used. Results for the several models showed various overall levels of turbulent viscosity, very different dynamic behavior in the flow patterns, variations in asymmetry as well as variations in predicted velocity. The standard $k \sim \varepsilon$ model showed the greatest turbulent viscosity, the SST $k \sim \omega$ model the least and the AKN low Re $k \sim \varepsilon$ model somewhat more than the $k \sim \omega$ model. The RSM results appeared to be diminishing in their unsteadiness toward a steady state as was seen in an earlier study.

Significant asymmetry is seen in the results for the standard $k \sim \varepsilon$ and RSM models and a lesser amount for the SST $k \sim \omega$ model while the AKN $k \sim \varepsilon$ results are generally quite symmetric. It has been recommended that asymmetry be built into the scaled model to prevent small and unidentified effects from causing asymmetry in a symmetric geometric model.

The issue of how dynamic is the actual flow should be resolved by experiment. However, the MIR data were taken at 2-3 frames/second, not nearly fast enough to even track vortex shedding. It is recommended to obtain data using a faster system to see how dynamic the actual flow really is, which will help sort out the turbulence modeling issue. It will also help to answer the question of whether there are multiple unstable flow patterns present that are complicating the ability of the CFD modeling to capture them. This will have ramifications regarding turbulent heat transfer which is directly related to the primary concerns regarding the hot helium flow in the lower plenum. That is, how hot are local hot spots and how good is the thermal mixing of the helium in the lower plenum?

\section{NOMENCLATURE}

$\begin{array}{ll}\text { CFD } & \text { computational fluid dynamics } \\ \text { INL } & \text { Idaho National Laboratory, Idaho Falls, ID } \\ k & \text { turbulent kinetic energy } \\ \text { MIR } & \text { matched index of refraction } \\ \text { NGNP } & \text { next generation nuclear plant } \\ \text { PIV } & \text { particle image velocimetry } \\ \text { RSM } & \text { Reynolds stress transport turbulence model } \\ \text { SST } & \text { shear stress transport } \\ <u> & \text { ensemble mean velocity, X direction } \\ U & \text { time mean velocity, X direction } \\ \text { URANS } & \text { unsteady Reynolds-averaged Navier-Stokes } \\ \text { VHTR } & \text { very high temperature reactor } \\ <w> & \text { ensemble mean velocity, Z direction } \\ W & \text { time mean velocity, Z direction } \\ y^{+} & \text {dimensionless wall distance } \\ \varepsilon & \text { turbulent energy dissipation rate } \\ \omega & \text { specific energy dissipation rate }\end{array}$

\section{ACKNOWLEDGMENTS}

This manuscript has been authored by Battelle Energy Alliance, LLC under Contract No. DE-AC07-05ID14517 with the U.S. Department of Energy.

\section{REFERENCES}

[1] H. M. McIlroy, D. M. McEligot and R. J. Pink, Measurement of Flow Phenomena in a Lower Plenum Model of a Prismatic Gas-Cooled Reactor, Proceedings of the $16^{\text {th }}$ International Conference on Nuclear Engineering (ICONE-16), Orlando, May 11-15, 2008.

[2] R. W. Johnson, Development of a CFD Analysis Plan for the First VHTR Standard Problem, Proceedings of the $4^{\text {th }}$ International Topical Meeting on High Temperature Reactor Technology (HTR2008), Washington, D. C., Sept. 28 - Oct. $1,2008$.

[3] FLUENT, version 6.3.26, 2008. FLUENT Inc., 10 Cavendish Court, Centerra Resource Park, Lebanon, NH, 03766.

[4] STAR-CCM+, version 3.06, 2008, CD-adapco, 60 Broadhollow Road, Melville, NY 11747.

[5] R. W. Johnson, CFD Investigation Of Experimental Data Proposed To Be A Validation Data Set, Proceedings of the 17th International Conference on Nuclear Engineering (ICONE17), paper ICONE17-75604, Brussels, Belgium, July 12-16, 2009.

[6] R. W. Johnson, "Modeling Strategies for Unsteady Turbulent Flows in the Lower Plenum of the VHTR," Nuclear Engineering \& Design, 238, pp. 482-491, 2008. 